\title{
A Structural Complexity Metric Method for Complex Information Systems
}

\author{
Aimin Luo ${ }^{1 *}$, Mengmeng Zhang ${ }^{1}$, Yi Mao², Yuxiao Kou' ${ }^{1}$, Xiaoxue Zhang ${ }^{1}$ \\ ${ }^{1}$ Science and Technology on Information Systems Engineering Laboratory, National University of Defense \\ Technology, Changsha, Hunan, 410073, P.R.China \\ ${ }^{2}$ State Key Laboratory of Air Traffic Management System and Technology, Nanjing, 210007, China \\ * Corresponding author. Tel.: +86 13786115986; email: amluo@nudt.edu.cn \\ Manuscript submitted February 15; accepted April 12, 2019. \\ doi: 10.17706/jsw.14.7.332-339
}

\begin{abstract}
A system structure is deemed as a trade-off between requirements and complexity. An effective measurement of structural complexity is the basis of choosing a reasonable system structure. In this paper, a structural complexity evaluation model of information systems is established through three factors: subsystem complexity, interactional complexity, and topological complexity. In addition, we introduce quantitative calculation methods related to the three factors. Finally, the proposed model is verified by the case of an air defense suppression system.
\end{abstract}

Key words: Structural complexity, subsystem complexity, interactional complexity, topological complexity, measurement.

\section{Introduction}

Basically, the design of information systems should be materialized as a simple enough system architecture under the premise of meeting system functions. To a certain extent, a complex information system raises the difficulties of system design and development, increases the development cost and time, and reduces the system reliability. Therefore, we argue that the measurement of system complexity is beneficial for controlling and managing the complexity during the information system design and development stages.

Currently, the complexities of information systems are inevitably increased with the widespread use of advanced information technologies, such as cloud computing, big data, mobile Internet, and the Internet of Things. The above technologies expand application areas of information systems, vary user number and types, and consequently demand more complicated functions. Additionally, the system complexity can also be increased by uncertain scenarios and tasks.

Therefore, system architects and designers should develop an optimized system architecture to balance system requirements and complexity. Which means, the designers must be able to reasonably analyze the complexity of different structures in the design phase. To achieve this goal, an appropriate measurement indicator should be introduced to measure system complexity.

Multiple complexity metrics have been proposed in the literature. In the software engineering field, the software complexity has been evaluated since the 1970s, with the help of indicators including use lines of code, algorithmic test paths, programming efforts, and information flows [1]-[6]. Some of the well-known metrics include the cyclomatic metric proposed by Mccabe [1], [6] and the interconnectivity-based metric developed by Kafura and Henry [2]. In addition, Brito [4] proposes seven standards for object-oriented 
software lifecycle and quality assurance. A set of formal theorems for evaluating the features of software metrics are proposed by Weyuker [5].

The system complexity is also studied in other engineering areas. For example, El-Haik and Yang [7] introduce a complexity metric method on the basis of Boltzmann derivation of entropy. Whitney et al. [8] focus on the relationships between components and their interfaces on the purpose of complexity metric. Kreimeyer et al. [9], [10] constrain the metric from the system hierarchy and information transfer efficiency. Additionally, Sinha et al. [11]-[14] suggest the use of matrix energy to evaluate structural complexity. With regard to the complex military information systems, Domerçant [16] proposes a structural complexity assessment method based on cyclomatic complexity. In order to manage the complexity of aerospace systems, Becz et al. [18] have developed an abstract complexity measure approach. Furthermore, Ref. [19] presents a complexity classification in the system design stage.

We mainly discuss the structural complexity analysis method of information systems in this paper. The rest of this paper is organized as follows. Section 2 analyzes the related research results of the complexity metric, and concludes a structural complexity measure model. To quantify this model, Section 3 proposes a detailed calculation method. Section 4 verifies the proposed method with an illustrative case.

\section{Structural Complexity Metric Model}

Recently, the complexity theory has attracted extensive attention within the field of system development. A variety of complexity classifications have been proposed. Ref. [19] divides the complexity of system design and development into three categories: 1) structural complexity; 2) dynamic complexity; and 3) organizational complexity. Specifically, the structural complexity is reflected by the composition and structure of the system. The dynamic complexity is determined by the system behaviors. Which means, the dynamic complexity can be raised by the difficulties of predicting and describing the behavioral processes. Furthermore, organizational complexity relates with the organizational structures and development processes. For the purpose of system architecture optimization design, we mainly focus on the structural complexity of information systems in this paper.

In general, the complexity of an information system is closely related to the system functions, subsystem connections, and subsystem topology. Detailly, the complexity can be positively influenced by the system functions and subsystems. In addition, the increasing interactions and requirements between subsystems can produce a higher complexity. Furthermore, the system topology can also differentiate the complexity, although with the same number of system functions and subsystem interactions. Overall, the structural complexity of information systems is a measurable characteristic, which depends on the quantity of subsystems and their connectivity structure.

According to the above explanation, the structural complexity metric is presented as formula (1):

$$
C=C 1+C 2 \times C 3
$$

The introduction of Eq. (1) considers several previous research on complexity measures [11], [16], [17]. According to Eq. (1), the structural complexity is composed by the sum of individual subsystem's complexity and the complexity arising from interconnections among subsystems. The structural complexity metric (C) shown in Eq. (1) includes three terms: $C 1$ refers to the sum of individual subsystem's complexities; $C 2$ is the sum of complexities arising from interactions between subsystems; $C 3$ represents the topological complexity of the system, depending by the interface deployment of the system.

C3 expresses the relationship between subsystems' connections and system complexity from another perspective. Different from $C 2$ focusing on the individual connection, $C 3$ aims to explain the global impact of 
subsystems' connections with various topology. One complicated topological structure can produce an extra complexity although with less subsystems' connections. Therefore, the complexity arising from interconnections between subsystems is formalized by the multiple of $C 2$ and $C 3$.

\section{Structural Complexity Metric}

\subsection{Complexity of Subsystem (C1)}

According to Eq.(1), the complexity of subsystems is an important factor affecting the overall complexity of information systems. $C 1$ in Eq.(1) can be calculated by the sum of the complexity of each subsystem, ie

$$
C 1=\sum_{i=1}^{N} c_{i}
$$

where $c_{i}$ represents the complexity of ith subsystem, $\mathrm{N}$ is the number of subsystems in an information system.

The complexity of each subsystem refers to its internal complexity, which is closely related to the functions it performs. The complexity is positively influenced by the functions and their executive processes.

We suppose that $\mathrm{F}=\left\{f_{1}, f_{2}, \cdots, f_{M}\right\}$ represents the functions performed by one system. Meanwhile, the complexity of each function is different. The complexity of one function can be determined by experts' experience. Assuming that the complexity of each function in set $\mathrm{F}$ is $\mathrm{w}_{1}, \cdots, w_{M}$, and subsystem $s_{i}$ can fulfill functions $\left\{f_{i_{1}}, \cdots, f_{n_{i}}\right\}$, we can determine the complexity of $s_{i}$ as following:

$$
c_{i}=\sum_{j=1}^{n_{i}} w_{j}
$$

Besides system functions, we argue that $\mathrm{C} 1$ is also related to the granularity of system decomposition. A subsystem that performs one function is less complex than another subsystem that performs multiple functions. Briefly, the finer the granularity of decomposition, the lower the complexity of each subsystem. Therefore, $C 1$ can be calculated by equation (3).

$$
C 1=\frac{\sum_{i=1}^{N} c_{i}}{N}=\frac{\sum_{i=1}^{N} \sum_{j=1}^{n_{i}} w_{j}}{N}
$$

\subsection{Interactional Complexity (C2)}

To express the complexity of interactions between subsystems, $C 2$ in Eq.(1) can be measured by Eq.(4)

$$
C 2=\sum_{i, j} \beta_{i j}
$$

where $\beta_{i j}$ denotes the complexity of interaction between subsystem $i$ and $j$.

In general, the interactional complexity is closely related to the interactional mode, content, and requirements. This kind of complexity can be calculated with the properties in the interactions of subsystems.

During the information exchanging process, the level of interoperability is a standard that describes how and what is required to interact between two objects. Levels of information systems interoperability (LISI) [21] classifies the interoperability levels according to the Procedure, Application Infrastructure and Data(PAID). According to LISI, the level of interoperability is divided into five levels, which reflect the interactional complexity from low to high. Therefore, the interoperability level is deemed as a measure of the interactional complexity $(C 2)$. 
In addition, the timeliness and confidentiality requirements of information interactions are also important factors affecting the complexity of interactions. Generally speaking, the timeliness of information interaction $\mathrm{T}$ is divided into three kinds, including real-time, near real-time, and non-real-time. At the same time, the level of confidentiality is generally divided into four levels, including top secret, confidential, secret, and public.

According to Eq.(4), $\beta_{i j}$ describes the complexity of information interactions between subsystem $s_{i}$ and $s_{j}$. Considering the above contents, the value of $\beta_{i j}$ can be represented by interoperability level $\left(I_{i j}\right)$, timeliness $\left(T_{i j}\right)$, and confidentiality $\left(S_{i j}\right)$. Therefore, $\beta_{i j}$ can be defined as following

$$
\beta_{i j}=\frac{I_{i j}}{5}+\frac{S_{i j}}{4}+\frac{T_{i j}}{3}
$$

We assume that matrix A denotes the adjacency matrix, which describes the interactional relationships among subsystems of information systems. $a_{i j}$ is an element of matrix A. And $i, j=1, \cdots N$.

$$
a_{i j}=\left\{\begin{array}{cc}
1 & n_{i} \rightarrow n_{j} \\
0 & \text { otherwaise }
\end{array}\right.
$$

Therefore, $C 2$ can be calculated by Eq.(6)

$$
C 2=\sum_{i=1}^{N} \sum_{j=1}^{N} \beta_{i j} a_{i j}
$$

\subsection{Topological Complexity}

In general, topological complexity can be measured by cyclomatic complexity or matrix energy. We will compare these two approaches and select a suitable method. The cyclomatic complexity is calculated on the basis of the system process model. According to the cyclomatic complexity metric, the topological complexity is expressed as Eq.7.

$$
C 3=e-n+2 p
$$

where $n=$ the number of vertices (nodes) in the process model, $e=$ the number of edges in the process model, and $p=$ the number of components that system partitions. We suppose $p=1$ in the paper.

According to Eq. (7), topological complexity is closely related to the missions or tasks performed by the system. The execution process of each task can result in the different system complexity. Due to that complex information systems usually perform multiple tasks, face with different scenarios, and even need to deal with uncertain tasks and scenarios, the cyclomatic complexity cannot accurately reflect the overall complexity of information systems.

With regards to complex information systems, the design of interactions between subsystems should consider the tasks accomplished by the system and combine the requirements of different scenarios. After analyzing the requirements of interactions in typical tasks and various scenarios, the system structure can be established to satisfy requirements of all tasks and scenarios. The structure is more comprehensive to reflect the system topology, also determines the uniqueness of complexity.

Ref.[20] calculates the cyclomatic complexity with system structure diagrams. However, this method can't explain the complex system topology perfectly. For example, Fig. 1 lists two system structures with the same number of nodes and edges. The connective structures in Figure 1 are different. According to Ref. [20], the cyclomatic complexities of these two structures are all $1(C 3(a)=C 3(b)=1)$. Considering the viewpoint of system tasks, their complexities should be obviously different. 


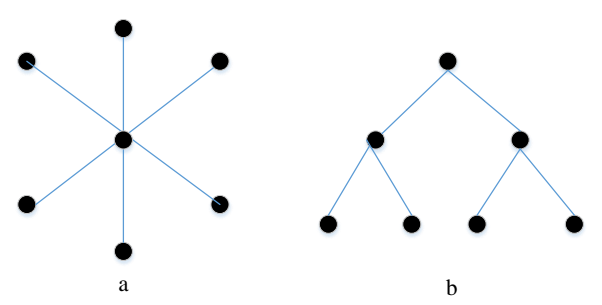

Fig. 1. Two structures of a system.

Matrix energy (denoted as $E$ ), which describes the singular value of the system adjacency matrix, is another measurement approach for interactional complexity. Taking Fig. 1 into consideration, their values of matrix energy are respectively $4.9(E(a))$ and $6.83(E(b))$. Which means, the different connective structures can result into different complexities.

Therefore, we adopt the matrix energy to measure the topological complexity. Specifically, the topological complexity is calculated by the singular value of the adjacency matrix of the system, as shown in Eq.8.

$$
C 3=E(A)=\sum_{i} \sigma_{i}
$$

where $E(A)$ refers to the sum of the singular values of matrix $A, \sigma_{i}$ represents the ith singular value of $A$.

\section{Case}

Considering the Suppression of Enemy Air Defenses (SEAD) system described in Ref. [17], we aim to analyze its structural complexity in this section Combining the system resources including sea, land, air, and space, the SEAD system mainly aims to weaken and destroy the capabilities of enemy's air defense systems.

The SEAD system is composed by F/A-18 Hornet, AH-64 Apache, EA-6B Prowler, M252 Mortar Crew, E-2 Hawkeye, and CVN. This system can perform functions including detecting, identifying, correlating/tracking, target assignment, and weapon controlling. The detailed activity flows and the mapping relationships between the activities and the subsystems are shown in Ref. [17].

The specific functions performed by subsystems are displayed in Table 1. Table 2 lists the complex degree of each function.

Table 1. The Functions Performed by Subsystems in SEAD

\begin{tabular}{|l|l|l|l|l|l|l|l|l|l|l|l|l|l|l|l|l|l|}
\hline Functions & 1.1 & 1.2 & 1.3 & 1.4 & 1.5 & 1.6 & 2.0 & 3.1 & 3.2 & 3.3 & 4.1 & 4.2 & 4.3 & 5.1 & 5.2 & 5.3 & 5.4 \\
\hline F/A-18 & & & & $\mathrm{X}$ & & & $\mathrm{X}$ & & $\mathrm{X}$ & & & & & $\mathrm{X}$ & & $\mathrm{X}$ & \\
\hline AH-64 & & & & $\mathrm{X}$ & & & $\mathrm{X}$ & & $\mathrm{X}$ & & & & & $\mathrm{X}$ & & $\mathrm{X}$ & \\
\hline EA-6B & & & & $\mathrm{X}$ & & & $\mathrm{X}$ & & $\mathrm{X}$ & & & & & & $\mathrm{X}$ & $\mathrm{X}$ & \\
\hline M252 & & & & & & & $\mathrm{X}$ & & $\mathrm{X}$ & & & & & $\mathrm{X}$ & $\mathrm{X}$ & & \\
\hline E-2 & & & & & $\mathrm{X}$ & $\mathrm{X}$ & & $\mathrm{X}$ & & $\mathrm{X}$ & $\mathrm{X}$ & & & & & & \\
\hline CVN & $\mathrm{X}$ & $\mathrm{X}$ & $\mathrm{X}$ & & $\mathrm{X}$ & $\mathrm{X}$ & & $\mathrm{X}$ & & & $\mathrm{X}$ & $\mathrm{X}$ & $\mathrm{X}$ & & & & $\mathrm{X}$ \\
\hline
\end{tabular}

Table 2. The Complexity of Functions

\begin{tabular}{|l|l|l|l|l|l|l|l|l|l|l|l|l|l|l|l|l|l|}
\hline Functions & 1.1 & 1.2 & 1.3 & 1.4 & 1.5 & 1.6 & 2.0 & 3.1 & 3.2 & 3.3 & 4.1 & 4.2 & 4.3 & 5.1 & 5.2 & 5.3 & 5.4 \\
\hline complexity & 1 & 1 & 1 & 1 & 1 & 1 & 1 & 1 & 1 & 1 & 1 & 1 & 1 & 1 & 1 & 1 & 1 \\
\hline
\end{tabular}

According to Eq.(3), $C 1$ is calculated as the following:

$$
C 1=\frac{\sum_{i=1}^{6} c_{i}}{6}=\frac{114}{6}=19
$$

We supposed that the attributes of the interactional relationship in the SEAD are shown in Table 1. Figure 
2 expresses the connective structure of SEAD [17]. According to Eq.(6), C2 can be obtained as 49.18.

Table 3. The Attributes of Interaction Relationships in SEAD

\begin{tabular}{|l|l|l|l|l|}
\hline Name & Interoperability & timeliness & confidentiality & $\beta_{i j}$ \\
\hline F/A-18 to CVN & 4 & 3 & 2 & 2.3 \\
\hline CVN to F/A-18 & 4 & 3 & 2 & 2.3 \\
\hline M252 to CVN & 4 & 3 & 2 & 2.3 \\
\hline CVN to M252 & 4 & 3 & 2 & 2.3 \\
\hline AH-64 to CVN & 4 & 3 & 2 & 2.3 \\
\hline CVN to AH-64 & 4 & 3 & 2 & 2.3 \\
\hline EA-6B to CVN & 4 & 2 & 2 & 1.97 \\
\hline CVN to EA-6B & 3 & 2 & 2 & 1.77 \\
\hline F/A-18 to E2 & 4 & 3 & 2 & 2.3 \\
\hline E2 to F/A-18 & 4 & 3 & 2 & 2.3 \\
\hline M252 to E2 & 4 & 2 & 2 & 1.97 \\
\hline E2 to M252 & 4 & 2 & 2 & 1.97 \\
\hline AH-64 to E2 & 4 & 3 & 2 & 2.3 \\
\hline E2 to AH-64 & 4 & 3 & 2 & 2.3 \\
\hline EA-6B to E2 & 4 & 2 & 2 & 1.97 \\
\hline E2 to EA-6B & 4 & 2 & 2 & 1.97 \\
\hline E2 to CVN & 4 & 2 & 2 & 1.97 \\
\hline CVN to E2 & 4 & 2 & 2 & 1.97 \\
\hline M252 to EA-6B & 3 & 2 & 2 & 1.77 \\
\hline EA-6B to M252 & 3 & 2 & 2 & 1.77 \\
\hline EA-6B to AH-64 & 3 & 2 & 2 & 1.77 \\
\hline AH-64 to EA-6B & 3 & 2 & 2 & 1.77 \\
\hline F/A-18 to EA-6B & 3 & 2 & 2 & 1.77 \\
\hline EA-6B to F/A-18 & 3 & 2 & 2 & \\
\hline
\end{tabular}

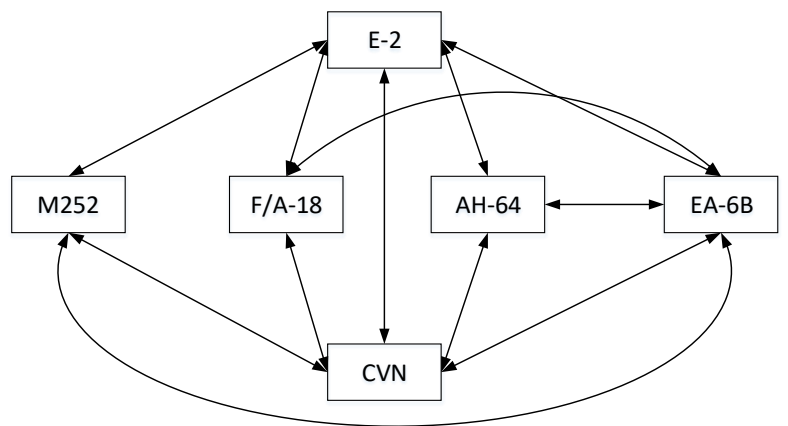

Fig. 2. the Structure of SEAD.

According to the structure of SEAD, the adjacency matrix A can be established as the following:

$$
A=\left(\begin{array}{llllll}
0 & 0 & 0 & 1 & 1 & 1 \\
0 & 0 & 0 & 1 & 1 & 1 \\
0 & 0 & 0 & 1 & 1 & 1 \\
1 & 1 & 1 & 0 & 1 & 1 \\
1 & 1 & 1 & 1 & 0 & 1 \\
1 & 1 & 1 & 1 & 1 & 0
\end{array}\right)
$$

The singular values of A are respectively 4.1623, 2.1623, 1.0, 1.0, 0.0, and 0.0. Therefore, C3 is 8.3246. According to Eq. (1), the structural complexity of SEAD is 428.4 .

$$
C=C 1+C 2 \times C 3=19+49.18 \times 8.3246 \approx 428.4
$$

Overall, with the proposed approach, this section analyzes the structural complexity of the SEAD system. The whole process measures the complexity simple and persuasive. The complexity result can be used to 
compare with other candidate structures in the design stage.

\section{Conclusion}

The structural complexity of information systems, which is an important factor affecting system capabilities, is related to subsystems and their interactions. In the system development stage, it is necessary to analyze the complexity of system structures, select a reasonable system architecture, and finally form an optimal system structure. Aiming at the design of information system, this paper proposed a measurement method of system structure complexity. This method focuses on the complexity of the interactions among subsystems, and can also be applied to analyze the structural complexity of system of systems.

\section{Acknowledgment}

This study is supported by the National Natural Science Foundation of China (No.71571189) and by State Key Laboratory of air Traffic Management System and Technology (No.SKLATM201806).

\section{References}

[1] Mccabe, T. J. (1976). A complexity measure. IEEE Trans. Software Engineering, 2(4), 308-320.

[2] Kafura, D., \& Henry, S. (1981). Software quality metrics based on inter-connectivity. System Software, 2(2), 121-131.

[3] Halstead, M. H. (1977). Elements of software science, operating and programming systems series. Elsevier, New York.

[4] Brito, F., \& Abreu, E. (1994). MOOD-metrics for object-oriented design. OOPSLA' 94 Workshop on Pragmatic and Theoretical Directions in Object-Oriented Software Metrics.

[5] Weyuker, E. J. (1988). Evaluating software complexity-measures. IEEE Transactions on Software Engineering, 14(9), 1357-1365.

[6] Watson, A., \& McCabe, T. (1996). Structured testing: A testing methodology using the cyclomatic complexity metric (NIST special publication 500-235). Gaithersburg: Computer Systems Laboratory National Institute of Standards and Technology.

[7] El-Haik, B., \& Yang, K. (1999). The components of complexity in engineering design. IIE Transactions, 31(10), 925-934.

[8] Whitney, D., Dong, Q., Judson, J., \& Mascoli, G. (1999). Introducing Knowledge-Based Engineering Into an Interconnected Product Development Process. ASME Paper No. DETC99/DTM-8741.

[9] Kreimeyer, M., \& Lindemann, U. (2011). Complexity metrics in engineering design: Managing the structure of design processes. Springer, Heidelberg, New York.

[10] Kortler, S., Kreimeyer, M., \& Lindemann, U. (2009). A planarity-based complexity metric. Proceedings of the International Conference on Engineering Design, Paper No. DS 58-6, Palo Alto, CA, Aug. 24-27.

[11] Sinha, K., \& De. W. O. (2013). Structural complexity quantification for engineered complex systems and implications on system architecture and design. ASME Paper No. DETC2013-12013.

[12] Sinha, K., \& De, W. O. L. (2013). A network-based structural complexity metric for engineered complex systems. Proceedings of the IEEE International on Systems Conference, Orlando, FL.

[13] Sinha, K. (2014). Structural complexity and its implications for design of cyber-physical systems. Ph.D. thesis, Massachusetts Institute of Technology, Cambridge, MA.

[14] Sinha, K., Shougarian, N. R., \& de De. W. O. (2017). Complexity management for engineered systems using system value definition. Complex Systems Design and Management, 155-170, New York: Springer.

[15] Tamaskar, S., Neema, K., \& DeLaurentis, D. (2014). Framework for measuring complexity of aerospace systems. Res Eng Des, 25(2), 125-137. 
[16] Sinha, K., \& Eun, S. S. (2018). Pareto-optimization of complex system architecture for structural complexity and modularity. Res Eng Design, 29, 123-141

[17] Domercant, J. C., \& Mavris, D. N. (2011). Measuring the architectural complexity of military systems-ofsystems. Proceedings of the IEEE Aerospace Conference.

[18] Becz, S., Pinto, A., Zeidner, L. E., Khire, R., Banaszuk, A., \& Reeve, H. M. (2010). Design system for managing complexity in aerospace systems. Proceedings of the 10th AIAA Aviation Technology, Integration, and Operations (ATIO) Conference.

[19] Braha, D., \& Bar, Y. Y. (2007). The statistical mechanics of complex product development: Empirical and analytical results. Manag Sci, 53:1127-1145.

[20] Malone, P., L. W. (2013). Measuring system complexity to support development cost estimates. Proceedings of IEEE Aerospace Conference.

[21] C4ISR Architectural Working Group. (1998). Levels of information systems interoperability (LISI). Retrieved March 7, 2011, from http://www.defencelink.mil/nii/org/cio/i3/lisirpt.p df

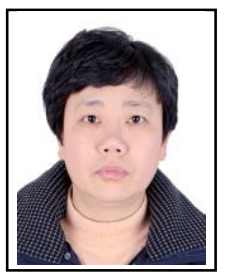

Aimin Luo was born in 1971. She received the M.Sc. degree and the Ph.D. degree from National University of Defense Technology (NUDT), Changsha, P. R.China. She is a professor of management science and engineering. Her current research interests include requirement engineering, military information system, enterprise architecture design and analysis.

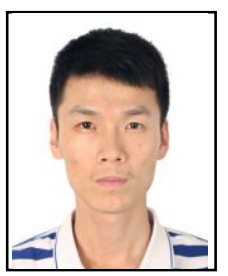

Mengmeng Zhang was born in 1990. He received the M.Sc. degree in management science and technology from NUDT in 2011. He is pursuing the Ph.D. degree in the School of System Engineering at NUDT. His research interests are information system architecture design, analysis and validation.

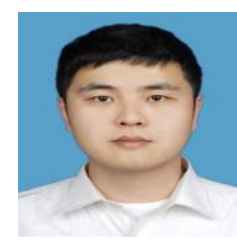

Yi Mao was born in China in 1985, received his Ph.D. degree in Nanjing University of Aeronautics and Astronautics. He is an engineer in state key laboratory of Air Traffic Management System and Technology, China. His research interests include transportation planning and management, information system architecture.

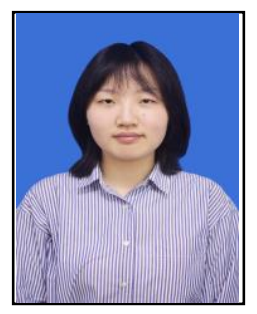

Yuxiao Kou was born in 1996. She received the bachelor of engineering degree in command information system engineering in 2017 from National University of Defense Technology, Changsha, China, where she is currently studying for a master's degree in management science and engineering. Her research is focused on analyzing and evaluating information systems.

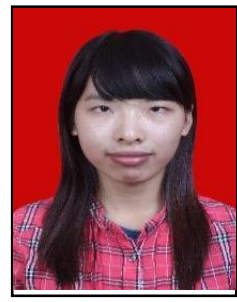

Xiaoxue Zhang was born in 1986. She received the Ph.D. degree in military science in 2014 from National University of Defense Technology (NUDT), Changsha, P. R.China. She is a lecturer of the School of System Engineering at NUDT. Her research interests are enterprise architecture design and analysis. 